\title{
Primary drug resistance among tuberculosis patients with diabetes mellitus: a retrospective study among 7223 cases in China
}

This article was published in the following Dove Press journal: Infection and Drug Resistance

Wan-mei Song, ${ }^{1,2, *}$ Yang Shao, ', Jinyue Liu, ${ }^{3}$ Ning-ning Tao, ${ }^{4}$ Yao Liu, ' Qianyun Zhang, ${ }^{1,2}$ Ting-ting $X_{u}$,' Shi-jin Li, ${ }^{1,2}$ Chun-Bao Yu, ${ }^{5}$ Lei Gao, ${ }^{6}$ Liangliang Cui, ${ }^{7,8}$ Yi-fan Li, ' Huai-chen $\mathrm{Li}^{1,9}$

'Department of Respiratory Medicine, Shandong Provincial Hospital Affiliated to Shandong University, Shandong Provincial Hospital Affiliated to Shandong First Medical University, Jinan 25002I, Shandong, People's Republic of China; ${ }^{2}$ Department of Clinical Medicine, Cheeloo College of Medicine, Shandong University, Jinan 2500 I2, Shandong, People's Republic of China; ${ }^{3}$ Department of Clinical Medicine, School of Medicine and Life Sciences, University of JinanShandong Academy of Medical Sciences, Jinan 250200, Shandong, People's Republic of China; ${ }^{4}$ Department of Clinical Medicine, Medical College, Graduate School of Peking Union Medical College, Beijing 100005, People's Republic of China; ${ }^{5}$ Department of Respiratory Medicine,

Shandong Provincial Chest Hospital, Jinan 2500 I3, Shandong, People's Republic of China; ${ }^{6}$ National Health Commssion Key Laboratory of Systems Biology of Pathogens, Institute of Pathogen Biology, and Center for Tuberculosis Research, Chinese Academy of Medical Sciences and Peking Union Medical College, Beijing I00730, People's Republic of China; ${ }^{7}$ Department of Environmental Health, Jinan Municipal Center for Disease

Control and Prevention, Jinan 25002I, Shandong, People's Republic of China; ${ }^{8}$ Department of Biostatistics, School of Public Health, Shandong University, Jinan 2500 I2, Shandong, People's Republic of China; ${ }^{9}$ Department of Chinese Medicine Integrated with Western Medicine, College of Traditional Chinese Medicine, Shandong University of Traditional Chinese Medicine, Jinan 250355, Shandong, People's Republic of China

*These authors contributed equally to this work

Correspondence: Yi-fan Li; Huai-chen Li Department of Respiratory Medicine Shandong Provincial Hospital Affiliated to Shandong University, Shandong Provincial Hospital Affiliated to Shandong First Medical University, 324 Jingwuweiqi Road, Huaiyin District, Jinan 25002I, People's Republic of

China

Tel +86 I 8663706873 ;

+86 I 3031737646

Email1035312289@qq.com;

lihuaichen@I63.com
Background: Given the high burden of tuberculosis (TB) and diabetes mellitus (DM) in China and the worse outcome of TB-DM cases (refers to TB patients with diabetes), and drug-resistant tuberculosis cases (DR-TB), it is of great significance to explore the association between diabetes and primary DR-TB for TB elimination target in China. We assessed the clinical characteristics, drug-resistance profile, and increased risk of resistance among TB-DM patients across China from 2004 to 2017.

Method: 7223 cases with drug-susceptibility data were collected from Shandong, China. Categorical baseline characteristics of new TB cases were compared by DM status using Fisher's exact or Pearson Chi-square test. Univariable analysis and multivariable logistic models were used to estimate the association between diabetes and different drug-resistance profiles and the risk factors of primary drug resistance among TB-DM cases.

Result: Of 7223 newly diagnosed TB patients, 426 (5.90\%) were TB-DM cases. TB-DM csaes were more likely to be older, accompanied by higher body mass index (BMI) and hypertension than TB-no DM cases (refers to TB patients without diabetes). The rates of DRTB (21.83\% vs $16.96 \%$ ), polydrug resistant TB (PDR-TB, $6.10 \%$ vs $3.80 \%$ ), isoniazid (INH) + streptomycin (SM)-resistant TB (4.93\% vs 3.13\%), and SM-resistant TB $(16.20 \%$ vs $11.7 \%$ ) among TB-DM group were higher than TB-no DM group, $P<0.05$. DM was significantly associated with any DR-TB (adjusted (aOR):1.30; 95\% CI, 1.02-1.65), SMrelated resistance (aOR: 1.43; 95\% CI, 1.08-1.88), PDR-TB (OR: 1.57; 95\% CI, 1.04-2.36; aOR: 1.59; 95\% CI, 1.04-2.44), compared with pan-susceptible TB patients $(P<0.05)$.

Conclusion: Our study indicated that TB-DM groups had a higher proportion of drug resistance than TB groups, and diabetes was identified as a risk factor of total DR, PDR, $\mathrm{SM}$ resistance and $\mathrm{INH}+\mathrm{SM}$ resistance among newly diagnosed TB cases. Good management of diabetes and TB infection screening program among DM patients might be necessary for improving TB control in China.

Keywords: tuberculosis, primary drug resistance, diabetes, risk factor

\section{Introduction}

Globally, there were approximately 10 million new tuberculosis (TB) cases and an estimated 400 million diabetes cases in 2017, and of which 558,000 were multidrugresistant TB (MDR-TB, defined as resistance to at least rifampicin and isoniazid) or rifampin-resistant TB (RR-TB). Both TB and diabetes mellitus (DM) were one of the top 10 causes of death, and TB was the leading cause of death from a single infectious agent (more than HIV/AIDS). ${ }^{1,2}$ Previous studies $^{3-5}$ had demonstrated that DM was a risk factor of TB infection, and there was a three-fold increased risk of TB infection 
among people with DM than those without. It was reported that the proportions of TB-DM (refers to TB patients with diabetes) cases among newly diagnosed TB patients in India, some Pacific islands, and Texas-Mexico border region were $54 \%, 40 \%$, and $25 \%$, respectively. ${ }^{6}$

Recently, epidemiological studies and whole-genome sequencing analysis of Mycobacterium tuberculosis provided evidence that the majority of drug-resistant tuberculosis cases (DR-TB) were due to primary infection of resistant TB strains, ${ }^{7,8}$ and whether concomitant diabetes was a risk factor of primary infection for DR-TB remained understudied. Researches in India, Spain, and Turkey had found that $10-23 \%$ of MDR-TB patients had concomitant diabetes. ${ }^{9}$ Due to global increases in MDR-TB and DM, ${ }^{1,2}$ it was a necessity to explore the relationship between diabetes and primary drug resistance of TB. ${ }^{9}$ Other studies ${ }^{10,11}$ have reported the association between diabetes and MDR$\mathrm{TB}$, but the results were controversial and their samples were not enough as well. In addition, there remains a paucity of information about the relationship between diabetes and various subgroups of primary DR-TB and clinical characteristics of TB-DM cases, especially in China, which had the second highest burden of TB in 2017. ${ }^{1}$

Given the high burden of TB and diabetes in China and the worse outcome of TB-DM cases and DR-TB cases, ${ }^{1,11}$ it is of significance to explore the association between diabetes and primary DR-TB for TB elimination target in China. Thus, the objectives of the present study were 1) to estimate the prevalence of TB-DM patients among new TB cases and DR-TB cases in Shandong, China, from 2004 to 2017 ; 2) to explore the clinical characteristics of newly diagnosed TB-DM patients compared with TB cases without DM (TB-no DM); 3) to investigate the increased risk of various primary drug resistance among TB-DM patients.

\section{Methods}

\section{Ethics statement}

Ethical approvals of our study were obtained from the Ethics Committee of Shandong Provincial Hospital, affiliated with Shandong University (SPH) and the Ethics Committee of Shandong Provincial Chest Hospital (SPCH), China. Before data collection, statistical analysis, and reporting, any personal identifiers of TB patients were removed by monitoring stations. Patient informed consents were not required, and this study was conducted in accordance with the Declaration of Helsinki.

\section{Setting and participants}

Between 1 January 2004 and 31 December 2017, a retrospective observational study of people who were newly diagnosed with TB was conducted in Shandong, China. Shandong, a coastal province of the East China region, consisted of 17 municipalities and 140 counties (districts) with 100 million inhabitants in 2018 . It is located at $36^{\circ} 24^{\prime}$ $\mathrm{N}$ latitude $118^{\circ} 24^{\prime} \mathrm{E}$ longitude with an area of 157,100 $\mathrm{km}^{2}$. According to a national survey of TBin 2010, there were 211,900 active pulmonary TB cases (range: 170,100 to 253,600 ), and TB prevalence was 271 per 100,000 among people aged $>15$ years in Shandong, China. ${ }^{12}$ The prevalence of diabetes, prediabetes, and previously diagnosed diabetes in Shandong was estimated to be $3.5 \%$, $6.0 \%$, and $1.2 \%$, respectively. ${ }^{13}$

This study involved 7223 new TB cases with drug susceptibility data and information on diabetes, of which $426(5.9 \%)$ TB cases suffered from diabetes. Two province-level hospitals (SPH and SPCH), 13 municipallevel, and 21 county-level local health departments were responsible for the surveillance of DR-TB in Shandong from 2004 to 2017. Sociodemographic and clinical characteristics on age, sex, body mass index (BMI), drinking, smoking, cavity, and extrapulmonary TB were collected by questionnaires. Patients infected with nontuberculosis mycobacteria (NTM), retreated TB cases, or patients without information on diabetes were excluded (Figure 1).

\section{Laboratory diagnosis and drug susceptibility testing}

Each surveillance site collected two sputum samples from all eligible patients, and then sent all sputum samples to the TB Reference Laboratory of Shandong Provincial Chest Hospital (SPCH). Further examinations including bacteriologic culture, drug susceptibility testing (DST), and species identification were performed in this laboratory. Isolates were inoculated into tubes of acidified Löwenstein-Jensen (L-J) medium after conventional pretreatment process. ${ }^{14}$ Cultures with growing colonies were sent for further identification and DST. Based on previous published protocol, ${ }^{15}$ standard traditional biochemical testings including P-nitrobenzoic acid, 2-thiophene carboxylic acid hydrazide testing, and 16S rRNA gene sequence analysis (MicroSeq ID Microbial Identification Software (version 2.0); Applied Biosystems, Foster City, CA, USA) ${ }^{16}$ were used to differentiate Mycobacterium tuberculosis from other Mycobacteria spp. 


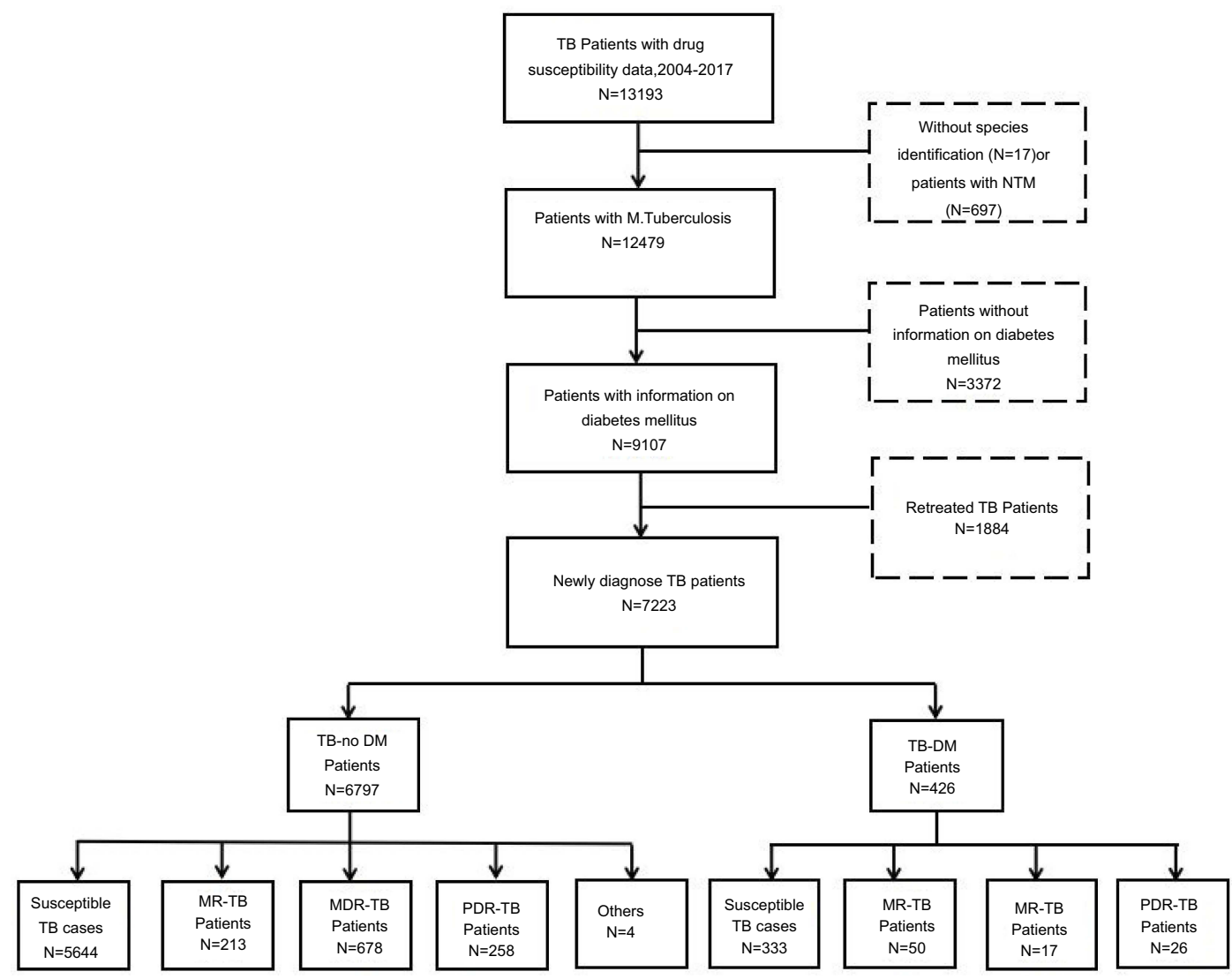

Figure I TB-DM patients and TB-no DM patients in Shandong, China. TB-no DM refers to TB patients without diabetes; others in TB-No DM refer to those which are DRTB but belong not to MR-TB, MDR-TB, and PDR-TB.

Abbreviations: NTM, nontuberculous mycobacteria; DR-TB, drug-resistant tuberculosis; MR-TB, monoresistant tuberculosis; MDR-TB, multiresistant tuberculosis; PDRTB, polydrug resistant tuberculosis; TB-DM refers to TB patients with diabetes.

The diagnosis of diabetes has been based on glucose criteria for decades, including the fasting plasma glucose (FPG) and the 75 -g oral glucose tolerance test (OGTT). ${ }^{17}$ The screening and laboratory diagnosis for DM strictly followed national guidelines, and an FPG was examined by venous plasma and biochemical analyzer with cutoff thresholds recommended by the WHO. ${ }^{17,18}$ Diagnostic criteria include the following: 1) $\mathrm{FPG} \geq 7.0 \mathrm{mmol} / \mathrm{L}(126 \mathrm{mg} / \mathrm{dl}) ; 2)$ A 2-hr plasma glucose level of $200 \mathrm{mg} / \mathrm{dL}(11.1 \mathrm{mmol} / \mathrm{L})$ or higher during OGTT. ${ }^{18}$

DST of Mycobacterium tuberculosis was performed using absolute concentration method on L-J media, and all procedures were carried out in accordance with the guideline of WHO. ${ }^{19}$ The concentrations of four first-line anti-TB drugs were as follows: $0.2 \mu \mathrm{g} / \mathrm{mL}$ (isoniazid, INH), $40 \mu \mathrm{g} / \mathrm{mL}$ (rifampin, RFP), $10 \mu \mathrm{g} / \mathrm{mL}$ (streptomycin, SM), and $2 \mu \mathrm{g} / \mathrm{mL}$ (ethambutol, EMB). DST of ethionamide, fluoroquinolone, kanamycin, and pyrazinamide was not routinely performed. Two professionally trained investigators were independently responsible for quality assessment and data extraction, and all laboratories involved in our study regularly accepted the external quality assessment of Superior TB National Reference laboratory in SPCH.

\section{Definitions ${ }^{20}$}

DR-TB is classified as having acquired or primary drug resistance on the basis of a history of previous treatment.

TB-DM and TB-no DM were defined as TB patients with or without diabetes, respectively.

Monoresistance (MR) refers to resistance to one firstline anti-TB drug only.

Polydrug resistance (PDR) refers to resistance to more than one first-line anti-TB drug, other than both isoniazid and rifampicin.

Multidrug resistance (MDR) refers to resistance to at least both isoniazid and rifampicin.

\section{Statistical analysis}

Categorical baseline characteristics of new TB cases including age $(0-14,15-24,25-44,45-64,65+)$, sex (male or female), BMI $(<18.5,18.5-24.9, \geq 25)$, drinking (yes/no), 
smoking (yes/no), cavity (yes/no), and patient type (extrapulmonary $\mathrm{TB} /$ pulmonary $\mathrm{TB}$ ) were compared by DM status using Fisher's exact or Pearson Chi-square test. Univariable analysis and multivariable logistic models were used to estimate the association between diabetes and different drug-resistance profile (for instance, DR-TB, MDR-TB, PDR-TB, SM-resistant TB, and SM+INH resistant TB). Similarly, logistic regression models were applied to explore the risk factors of primary drug resistance among TB-DM cases as well. Covariates adjusted for multivariable models were chosen based on previous studies. ${ }^{4,11}$ Factors including age, sex (male or female), BMI, drinking (yes/ no), smoking (yes/no), cavity (yes/no)m and hypertension (yes/no) were adjusted for multivariable logistic regression model. All statistical analyses were performed using SPSS software (version 20.0). A two-sided $P$-value $<0.05$ was considered statistically significant.

\section{Results}

\section{Study cohort and baseline characteristics}

During the study period, a total of 7223 newly diagnosed TB patients with drug susceptibility data and information on diabetes were included; 6797 (94.10\%) were TB-no DM cases and $426(5.90 \%)$ were TB-DM cases. The mean age of TB cases $(48.76 \pm 20.13$ years) was younger than TB-DM cases (55.66 \pm 11.33 years) $(P<0.001)$. Both these two groups had more males than females, and the proportion of male cases was $83.51 \%$ (5676/6797) and 86.38\% (368/426), respectively $(P>0.01)$. The age distribution of TB-no DM cases and TBDM cases showed about $0.28 \%$ and $0.00 \%$ under age 14 , $17.12 \%$ and $2.12 \%$ between the ages of 15 and 24 years, $26.07 \%$ and $18.63 \%$ between 25 and 44 years, $31.50 \%$ and $51.18 \%$ between the ages of 45 and 64 years, and $25.03 \%$ and $28.05 \%$ aged more than 65 years, respectively. Moreover, TBDM group had more cases aged between 25-44 and 45-64 years, and less cases between 15 and 24 years than TB-no DM group $(P<0.001)$. The percentage of cavity (62.05\%), smokers (33.68\%), and drinkers (29.71\%) among TB-DM cases was higher than that among TB-no DM cases $(42.43 \%, 25.83 \%$, $21.95 \%$, respectively) $(P<0.01)$. There were no significant differences in types (extrapulmonary TB/pulmonary TB) and some comorbidities including asthma $(0.50 \%$ vs $0.70 \%)$, COPD $(2.24 \%$ vs $2.58 \%)$, bronchiectasis $(0.40 \%$ vs $0.47 \%)$, silicosis $(0.21 \%$ vs $0.00 \%)$, and cancer $(0.25 \%$ vs $0.24 \%)$ between TB-no DM group and TB-DM group $(P>0.05)$. Furthermore, we found that TB-DM cases were more likely to be accompanied by hypertension ( $7.04 \%$ vs $1.21 \%$ ),
$P<0.001$. The average BMI of TB-no DM group and TBDM group was $20.34 \pm 3.26$ and $21.51 \pm 3.09$, respectively $(P>0.05)$, and the latter group had more cases between 18.5 and 24.9 (70.15\% vs $74.65 \%)$ or $\geq 25(4.60 \%$ vs $10.80 \%)$ but less cases $<18.5$ (25.25\% vs $14.55 \%$ ), $P<0.05$ (Table 1 ).

\section{Drug-resistance profile}

As shown in Table 2 and Figure S1, all these patients enrolled in our study were available for DST results, and the rates of DR-TB, PDR-TB, SM-resistant TB, INH+SM resistant TB among TB-DM group were higher than TB-no DM group $(P<0.05)$. Among 6797 TB-no DM cases and 426 TB-DM cases, $16.96 \%$ and $21.83 \%$ were DR-TB $(P<0.05)$; $9.97 \%$ and $11.74 \%$ were MR-TB $(P>0.05)$ including SM $(5.68 \%$ vs $7.75 \%, P>0.05)$, INH $(3.18 \%$ vs $3.05 \%, P>0.05)$, RFP (0.85\% vs $0.94 \%, P>0.05)$, EMB (0.19\% vs $0.00 \%) ; 3.13 \%$ and $3.99 \%$ were MDR-TB $(P>0.05)$ including MDR1 (INH +RFP, $0.68 \%$ vs $1.41 \%, P>0.05)$, MDR2 (INH+RFP+EMB +SM, $0.66 \%$ vs $1.41 \%, P>0.05$ ), MDR3 (INH+RFP+SM, $1.62 \%$ vs $1.17 \%, P>0.05$ ); and $3.80 \%$ and $6.10 \%$ were PDRTB $(P<0.05)$ including PDR1 (INH+EMB, $0.10 \%$ vs $0.23 \%$, $P<0.05$ ), PDR2 (INH+SM, $3.13 \%$ vs $4.93 \%, P>0.05$ ), PDR3 (RFP+SM, $0.29 \%$ vs $0.94 \%, P>0.05$ ), respectively. In addition, the proportion of any resistance to first-line drugs $(\mathrm{INH}$, RFP, EMB, SM) was $9.70 \%$ vs $12.21 \%$ ( $P>0.05), 4.38 \%$ vs $5.87 \%(P>0.05), 1.34 \%$ vs $1.64 \%(P>0.05)$, and $11.70 \%$ vs $16.20 \%(P<0.01)$ among TB-no DM group and TB-DM group, respectively.

\section{Risk factors of primary drug resistance among TB-DM cases}

Among the 426 TB-DM patients with available DST results, $333(79.17 \%)$ were fully susceptible, and 93 patients $(21.83 \%)$ had any resistance. In univariate analysis, TB-DM patients who were male (OR: 1.08; 95\% CI, 0.55-2.14), or with higher BMI (18.5-24.9, OR: 1.58; 95\% CI, 0.76-3.26; or $\geq 25$, OR: 1.27 ; 95\% CI, 0.47-3.42), smoking history (OR: 1.13; 95\% CI, 0.67-1.88), and alcohol use (OR: 1.33; 95\% CI, 0.79-2.25) had a higher risk of primary drug resistance. In contrast, older age (25-44 years, OR: 0.55 ; 95\% CI, $0.12-2.42$; or $45-64$ years, OR: $0.60 ; 95 \%$ CI, $0.15-2.48$, or $\geq 65$ years, OR: 0.48 ; 95\% CI, 0.11-2.06), cavity (OR: 0.83; 95\% CI, 0.51-1.35), and hypertension (OR: 0.73; 95\% CI, 0.06-9.16) were protective factors of primary drug resistance. However, none of those were statistically significant $(P>0.05)$. The results of the multivariable analysis were in accordance with the above except for smoking factor (Table 3). 
Table I Baseline characteristics of 7223 newly diagnosed adult TB patients in Shandong, China, 2004-20I7

\begin{tabular}{|c|c|c|c|}
\hline Characteristics & TB patients without diabetes $n=6797$ & TB patients with diabetes $n=426$ & $P$-value \\
\hline \multicolumn{4}{|c|}{ Age (years) $(n=6759 / n=424)$} \\
\hline Average & $48.76 \pm 20.13$ & $55.66 \pm 11.33$ & $P<0.00 I^{* *}$ \\
\hline $0-14$ & $19 / 6759(0.28 \%)$ & $0 / 424(0.00 \%)$ & 0.62 \\
\hline $15-24$ & $1157 / 6759(17.12 \%)$ & $9 / 424(2.12 \%)$ & $P<0.00 I^{* *}$ \\
\hline $25-44$ & $1762 / 6759(26.07 \%)$ & $79 / 424(18.63 \%)$ & $P<0.00 I^{* *}$ \\
\hline $45-64$ & $2129 / 6759(31.50 \%)$ & $217 / 424(51.18 \%)$ & $P<0.00 I^{* *}$ \\
\hline$>65$ & $1692 / 6759(25.03 \%)$ & I I 9/424 (28.07\%) & 0.16 \\
\hline \multicolumn{4}{|c|}{$\operatorname{Sex}(n=6797 / n=426)$} \\
\hline Female & $1|2| / 6797$ (16.49\%) & $58 / 426(13.62 \%)$ & 0.12 \\
\hline Male & $5676 / 6797(83.51 \%)$ & $368 / 426(86.38 \%)$ & 0.12 \\
\hline \multicolumn{4}{|c|}{ BMI $(n=6217 / n=426)$} \\
\hline Average & $20.34 \pm 3.26$ & $21.51 \pm 3.09$ & 0.85 \\
\hline$<18.5$ & |570/62I7 (25.25\%) & $62 / 426(14.55 \%)$ & $P<0.00 I^{* *}$ \\
\hline $18.5-24.9$ & $4361 / 6217(70.15 \%)$ & $318 / 426(74.65 \%)$ & $0.049 *$ \\
\hline$\geq 25$ & $286 / 6217(4.60 \%)$ & $46 / 426(10.80 \%)$ & $P<0.00 I^{* *}$ \\
\hline \multicolumn{4}{|c|}{ Cavity $(n=5887 / n=390)$} \\
\hline No & $3389 / 5877$ (57.57\%) & | $48 / 390(37.95 \%)$ & $P<0.00 I^{* *}$ \\
\hline Yes & $2498 / 5877(42.43 \%)$ & $242 / 390(62.05 \%)$ & $P<0.00 I * *$ \\
\hline \multicolumn{4}{|c|}{ Smoking $(n=526 \mathrm{I} / \mathrm{n}=380)$} \\
\hline No & $3902 / 526 I(74.17 \%)$ & $252 / 380(66.32 \%)$ & $0.00 \mathrm{I} * *$ \\
\hline Yes & |359/526| (25.83\%) & $128 / 380(33.68 \%)$ & $0.00 I * *$ \\
\hline \multicolumn{4}{|c|}{ Drinking $(5229 / n=377)$} \\
\hline No & $4081 / 5299$ (78.05\%) & $265 / 377$ (70.29\%) & $P<0.001 * *$ \\
\hline Yes & II 48/5299 (21.95\%) & I I2/377 (29.7I\%) & $P<0.00 I^{* *}$ \\
\hline \multicolumn{4}{|c|}{ Type $(n=6797 / n=426)$} \\
\hline Extrapulmonary TB & $32 / 6759(0.005 \%)$ & $\mathrm{I} / 426(0.24 \%)$ & 0.72 \\
\hline Pulmonary TB & $6765 / 6759(0.995 \%)$ & $425 / 426(99.76 \%)$ & 0.72 \\
\hline \multicolumn{4}{|c|}{ Comorbidities $(n=6797 / n=426)$} \\
\hline Asthma & $34 / 6759(0.50 \%)$ & $3 / 426(0.70 \%)$ & 0.48 \\
\hline COPD & I52/6759 (2.24\%) & I I/426 (2.58\%) & 0.64 \\
\hline Bronchiectasis & $27 / 6759(0.40 \%)$ & $2 / 426(0.47 \%)$ & 0.69 \\
\hline Silicosis & $14 / 6759(0.21 \%)$ & $0 / 426(0.00 \%)$ & 1.00 \\
\hline Hypertension & $83 / 6759(1.22 \%)$ & $30 / 426(7.04 \%)$ & $P<0.001 * *$ \\
\hline Cancer & $17 / 6759(0.25 \%)$ & $\mathrm{I} / 426$ (0.24\%) & 1.00 \\
\hline
\end{tabular}

Note: $* * P<0.01 ; * P<0.05$.

Abbreviation: BMI, body mass index.

The association between DM and primary drug-resistance profile

Table 4 illustrates the association between DM and primary anti-TB drug resistance. In univariate analysis, DM was significantly associated with PDR-TB (OR: $1.57 ; 95 \% \mathrm{CI}$, 1.04-2.36), but not with any DR-TB (OR: 1.195; 95\% CI,
0.944-1.513), INH-related resistance (OR: 1.179; 95\% CI, 0.87-1.60), RFP-related resistance (OR: 1.29; 95\% CI, 0.861.96), SM-related resistance (OR: $1.28 ; 95 \%$ CI, 0.98-1.67), INH+SM resistance (OR: 1.50; 95\% CI, 0.94-2.37), MR-TB (OR: 1.06; 95\% CI, 0.78-1.44), and MDR-TB (OR: 1.19; 95\% CI, 0.72-1.97), compared with patients with susceptible 
Table 2 Drug-resistant profile among TB-DM patients and TB-No DM patients

\begin{tabular}{|c|c|c|c|}
\hline Drug resistance & $\begin{array}{l}\text { DR-TB patients without diabetes } \\
n=\mid 153 / 6797(16.96 \%)\end{array}$ & $\begin{array}{l}\text { DR-TB patients with diabetes } \\
n=93 / 426(21.83 \%)\end{array}$ & $P$-value \\
\hline DR-TB (total) & $1153(16.96)$ & $93(21.83)$ & $0.01 *$ \\
\hline Any resistance to first-line drug & & & \\
\hline $\mathrm{INH}$ & $659(9.70)$ & $52(12.21)$ & 0.09 \\
\hline RFP & $298(4.38)$ & $25(5.87)$ & 0.15 \\
\hline EMB & 91 (I.34) & $7(1.64)$ & 0.60 \\
\hline SM & $795(11.70)$ & $69(16.20)$ & $0.005^{* *}$ \\
\hline MR TB (total) & $678(9.97)$ & $50(11.74)$ & 0.24 \\
\hline INH & $216(3.18)$ & $13(3.05)$ & 0.89 \\
\hline RFP & $58(0.85)$ & $4(0.94)$ & 0.78 \\
\hline EMB & $13(0.19)$ & $0(0.00)$ & 1.00 \\
\hline SM & $386(5.68)$ & $33(7.75)$ & 0.08 \\
\hline Others & $5(0.07)$ & $0(0.00)$ & 1.00 \\
\hline MDR (total) & $213(3.13)$ & $17(4.00)$ & 0.33 \\
\hline MDRI: INH+RFP & $46(0.68$ & $6(I .4 I)$ & 0.08 \\
\hline MDR2: INH+RFP+EMB+SM & $45(0.66)$ & $6(1.4 I)$ & 0.07 \\
\hline MDR3: INH+RFP+SM & $110(1.62)$ & $5(1.17)$ & 0.48 \\
\hline Others & $12(0.18)$ & $0(0.00)$ & 1.00 \\
\hline PDR & $258(3.80)$ & $26(6.10)$ & $0.02^{*}$ \\
\hline PDRI: INH+EMB & $7(0.10)$ & I $(0.23)$ & 0.39 \\
\hline PDR2: INH+SM & $213(3.13)$ & $21(4.93)$ & $0.04 *$ \\
\hline PDR3: RFP+SM & $20(0.29)$ & $4(0.94)$ & 0.05 \\
\hline Others & $18(0.26)$ & $0(0.00)$ & 0.62 \\
\hline
\end{tabular}

Note: $* P<0.05 ; * * P<0.01$.

Abbreviations: TB, tuberculosis; DR-TB, drug-resistant tuberculosis; MR-TB, monoresistant tuberculosis; MDR-TB, multiresistant tuberculosis; PDR-TB, polydrug resistant tuberculosis; EMB, ethambutol; INH, isoniazid; RFP, rifampin; SM, streptomycin.

strains. When analyzed in multivariable logistic regression model, we found that DM was significantly associated with any DR-TB (adjusted OR (aOR): 1.30; 95\% CI, 1.02-1.65), SM-related resistance (aOR: 1.43; 95\% CI, 1.08-1.88), PDR-TB (aOR: 1.59; 95\% CI, 1.04-2.44), compared with pan-susceptible TB patients $(P<0.05)$.

\section{Discussion}

Our findings provided an account of the high prevalence of DM among new TB cases, the differences in clinical characteristics between TB-DM cases and TB-no DM cases, the drug-resistance profile classified by DM, and the increased RR of primary drug resistance among TB-DM cases in China. This retrospective observational research among 7223 TB cases in China revealed that DM was a risk factor for primary drug resistance including any DR-TB, SMrelated resistance, and PDR-TB among newly diagnosed TB cases compared to pan-susceptible TB cases. Our findings suggested that diabetes may make people become more susceptible to MDR-TB strains, thus resulting in a higher risk of primary drug resistance.

We observed that the percentage of TB-DM among total TB cases in Shandong, China, was 5.90\%, and a meta-analysis on 84 studies from 31 countries found that the ratio of DM among TB patients in various low-income and middle-income countries varied from $1.80 \%$ to $45 \%$, with the majority $(n=44)$ between $10 \%$ and $30 \%{ }^{21}$ It was widely accepted that DM was a major risk factor of TB infection as a result of a weakened immune system, ${ }^{4-6,22}$ for example, DM patients tended to be accompanied with abnormally regulated cytokine responses to Mycobacterium tuberculosis. ${ }^{23}$ However, potential biological mechanisms remain largely unknown. ${ }^{22,23}$ A study of 6276 active TB cases in Australia found that the crude RR and adjusted RR of TB among all people with DM were 1.78 (95\% CI: 1.17-2.73) and 1.48 (95\% CI: 1.04-2.10), respectively. ${ }^{24}$ It was estimated that stopping the rise of diabetes would avoid 6 million (95\% CI: 5.1-6.9) incident cases in 13 countries with high TB burden during 20 years, and the diabetes epidemiology could substantially affect TB epidemic 
Table 3 Univariable and multivariable analysis of risk factors for DR-TB in patients with diabetes

\begin{tabular}{|c|c|c|c|c|c|c|}
\hline \multirow[t]{2}{*}{ Characteristics } & \multirow[t]{2}{*}{ Non-DR n=333(\%) } & \multirow[t]{2}{*}{ DR-TB $n=93(\%)$} & \multicolumn{2}{|c|}{ Univariable analysis } & \multicolumn{2}{|c|}{ Multivariable analysis } \\
\hline & & & OR $(95 \% \mathrm{Cl})$ & $P$-value & OR $(95 \% \mathrm{CI})$ & $P$-value \\
\hline \multicolumn{7}{|l|}{ Age (years) } \\
\hline $15-24$ & $6(1.80)$ & $3(3.23)$ & Reference & Reference & Reference & Reference \\
\hline $25-44$ & $62(18.62)$ & $17(18.28)$ & $0.55(0.12-2.42)$ & 0.43 & $0.48(0.11-2.20)$ & 0.35 \\
\hline $45-64$ & $167(50.15)$ & $50(53.76)$ & $0.60(0.15-2.48)$ & 0.48 & $0.53(0.12-2.24)$ & 0.38 \\
\hline$\geq 65$ & $96(28.83)$ & $23(24.73)$ & $0.48(0.11-2.06)$ & 0.32 & $0.43(0.10-1.89)$ & 0.26 \\
\hline \multicolumn{7}{|l|}{ Sex } \\
\hline Female & $46(13.8 I)$ & $12(12.90)$ & Reference & Reference & Reference & Reference \\
\hline Male & $287(86.19)$ & $81(87.10)$ & $1.08(0.55-2.14)$ & 0.82 & $1.01(0.49-2.09)$ & 0.97 \\
\hline \multicolumn{7}{|l|}{ BMI } \\
\hline$<18.5$ & $52(15.62)$ & $10(10.75)$ & Reference & Reference & Reference & Reference \\
\hline $18.5-24.9$ & $244(73.27)$ & $74(79.5 \%)$ & $1.58(0.76-3.26)$ & 0.22 & $1.53(0.72-3.24)$ & 0.27 \\
\hline$\geq 25$ & $37(11.11)$ & $9(9.68)$ & $1.27(0.47-3.42)$ & 0.64 & $1.12(0.38-3.23)$ & 0.84 \\
\hline \multicolumn{7}{|l|}{ Cavity } \\
\hline No & $112(33.63)$ & $36(38.7 I)$ & Reference & Reference & Reference & Reference \\
\hline Yes & $191(57.36)$ & $51(54.84)$ & $0.83(0.5 \mathrm{I}-1.35)$ & 0.46 & $0.80(0.49-1.33)$ & 0.39 \\
\hline \multicolumn{7}{|l|}{ Smoking } \\
\hline No & $200(60.06)$ & $52(55.9)$ & Reference & Reference & Reference & Reference \\
\hline Yes & $99(29.73)$ & $29(31.18)$ & $1.13(0.67-1.88)$ & 0.65 & $0.79(0.38-1.65)$ & 0.53 \\
\hline \multicolumn{7}{|l|}{ Drinking } \\
\hline No & $212(63.66)$ & $53(56.99)$ & Reference & Reference & Reference & Reference \\
\hline Yes & $84(25.23)$ & $28(30.11)$ & $1.33(0.79-2.25)$ & 0.28 & $1.56(0.74-3.30)$ & 0.24 \\
\hline \multicolumn{7}{|l|}{ Comorbidities } \\
\hline COPD & $9(2.70)$ & $2(2.15)$ & $0.67(0.04-10.25)$ & 0.77 & - & - \\
\hline Hypertension & $22(6.6 \mathrm{I})$ & $8(8.60)$ & $0.73(0.06-9.16)$ & 0.81 & $0.659(0.05-8.66)$ & 0.75 \\
\hline
\end{tabular}

Abbreviations: BMI, body mass index; COPD, chronic obstructive pulmonary disease.

in high burden countries. ${ }^{25}$ Compared to those without DM, TB-DM cases in China were older with higher BMI and higher proportion of smokers and drinkers and were more likely to have cavities, in accordance with previous study in Indonesia, Peru, Romania, and South Africa. ${ }^{4}$ Our findings were consistent with previous studies that both age ${ }^{26}$ and obesity ${ }^{27}$ were risk factors for DM among TB cases, but in contrast with findings ${ }^{28}$ that obesity was actually not identified as a risk factor of DM among TB patients because a majority of the population with $\mathrm{TB}$ in their study were undernourished, regardless of their diabetes status.There were no statistical significant associations between primary drug resistance and some social demographic factors or clinical characteristics including age (stratified into 0-14, 15-44, 45-64, 65+), BMI (stratified into $<18.5,18.5-24.9, \geq 25$ ), smoking, drinking, and cavity among 426 TB-DM patients, and few previous studies had focused on risk factors of drug resistance among TB-DM group. ${ }^{11}$

Previous researches had produced inconsistent results on the association between increased prevalence or risk of primary DR-TB with diabetes. ${ }^{10,11}$ Unlike our findings that TB-DM patients had a higher risk of any DR-TB, SM-related resistance, PDR-TB, but not MDR-TB, some retrospective cohort studies found that DM was significantly associated with MDR-TB in Caucasian (OR: 2.26, 95\% CI: 1.66-3.07), ${ }^{11}$ Asian (OR: 1.40, 95\% CI: 1.01-1.95) subgroups, ${ }^{11}$ and in Georgia (aOR: 2.27). ${ }^{29}$ Another study of 507 TB patients included 183 with coexistence of type 2 DM (T2 DM) and revealed that T2 DM patients had higher odds of resistance to INH (OR: 3.9, 95\% CI: 2.01, 7.9), 
Table 4 Association between diabetes and primary anti-tuberculosis drug resistance

\begin{tabular}{|c|c|c|c|c|}
\hline \multirow[t]{2}{*}{ Type } & \multicolumn{2}{|c|}{ Univariable analysis } & \multicolumn{2}{|c|}{ Multivariable analysis } \\
\hline & OR (95\%Cl) & $P$-value & aOR $(95 \% \mathrm{Cl})$ & $P$-value \\
\hline Any DR-TB & $\mathrm{I} .20(0.94-I .5 \mathrm{I})$ & 0.14 & $1.30(1.02-1.65)$ & $0.04 *$ \\
\hline INH-related resistance & $1.18(0.87-1.60)$ & 0.28 & $1.24(0.92-1.69)$ & 0.16 \\
\hline RFP-related resistance & $1.29(0.86-1.96)$ & 0.22 & $1.45(0.94-2.23)$ & 0.09 \\
\hline SM-related resistance & $1.28(0.98-1.67)$ & 0.07 & $1.43(1.08-1.88)$ & $0.01 *$ \\
\hline $\mathrm{INH}+\mathrm{SM}$ resistance & $1.50(0.94-2.37)$ & 0.09 & $1.53(0.95-2.44)$ & 0.08 \\
\hline MR-TB & $1.06(0.78-I .44)$ & 0.70 & $1.17(0.86-1.60)$ & 0.32 \\
\hline PDR-TB & $1.57(1.04-2.36)$ & $0.03 *$ & $1.59(1.04-2.44)$ & $0.03 *$ \\
\hline MDR-TB & $1.19(0.72-1.97)$ & 0.50 & $1.36(0.81-2.28)$ & 0.25 \\
\hline Pan susceptible & Reference & Reference & Reference & Reference \\
\hline
\end{tabular}

Note: $* p<0.05$

Abbreviations: DR-TB, drug-resistant tuberculosis; MR-TB, monoresistant tuberculosis; MDR-TB, multiresistant tuberculosis; PDR-TB, polydrug resistant tuberculosis; $\mathrm{INH}$, isoniazid; RFP, rifampin; SM, streptomycin.

RFP (OR: 3.4, 95\% CI: 1.6, 7.2) and pyrazinamide (OR: 9.4, 95\% CI: 2.8, 25.6), and TB-T2DM patients (vs TB-no T2DM patients) were more likely to present with MDR TB (HR: $3.1 ; 95 \%$ CI: $1.7,5.8 ; p<0.001) .{ }^{10}$ However, these previous studies above put new and relapse TB cases together to estimate the OR of MDR among TB-DM patients (vs TB-no DM patients); thus, a confusing impact of diabetes on primary and acquired drug resistance among TB groups may be exhibited, most of which also failed to distinguish the effects on different first-line anti-TB drugs either.

A 1:1 matched case-control study (146 pairs of participants) conducted in Henan, China, found that primary MDR-TB cases were more likely to suffer from diabetes, cardiovascular disease or other respiratory diseases, or cancer (aOR: 57.1; 95\% CI, 8.6-424.2). However, this study categorized diabetes with other diseases as general comorbidity factors; thus, the association between diabetes and primary MDR-TB was indeterminate. ${ }^{30}$ Our study had explored the association of DM and various primary DRTB cases separately, and to some extent, it made up the scarcity of former researches ${ }^{4,10,11}$ in this topic. Studies in model organisms indicated that drug-resistant TB strains were often associated with a fitness cost (refers to the lower virulence, infectivity, reproductive capacity, and less transmission than the drug-susceptible strains), ${ }^{31,32}$ while diabetes usually contributed to an altered immune function which may result in an increased susceptibility to bacterial infections such as $\mathrm{TB},{ }^{33}$ but whether impaired immune system could lead to a higher risk of primary MDR-TB or other drug resistance needs further investigation. In some studies, MDR-TB strains had been associated with less ongoing transmission than sensitive strains. $^{32,34}$ For instance, a 3-year prospective cohort household follow-up study in South Lima and Callao, Peru, which measured the incidence of TBdisease among 1055 household contacts of 213 MDR-TB index cases and 2362 household contacts of 487 drug-susceptible index cases, demonstrated that MDR-TB was less transmissible than drug-susceptible TB, at least in households. ${ }^{32}$ A low relative fitness of MDR-TB strains improves the possibilities of containing and diminishing the transmission of DR-TB strains. ${ }^{32}$ Researches in animal models ${ }^{35,36}$ and some molecular epidemiological studies ${ }^{31,32,37}$ by measuring the proportion of strains that were genetically clustered and attributable to recent transmission found that partial DR-TB strains were less pathogenic, whilst diabetes patients were more susceptible to developing infections than normal population due to immune system defenses and some diabetes-related health issues, such as nerve damage and reduced blood flow to the extremities. ${ }^{38}$ However, more biological mechanisms and characteristics in genomics that could make a further explanation on how diabetes can influence the transmission of various types of DR-TB strains need further investigating.

This study has many strengths. First, total DR-TB patients had been stratified into various subgroups such as MDR-TB, MR-TB, PDR-TB, SM-resistant TB, and INH-resistant TB, which would make it possible for us to investigate the association between diabetes and the transmission of TB strains with different antibiotic resistance mutations. Second, our study had involved overall newly diagnosed TB cases with DM information and DST results in Shandong, China, from 2004 to 2017, 
leading to a high reliability and validity of our results. This study also has several limitations. First, the diagnosis of DM was based on medical records of TB patients; therefore, it may lead to an information bias. Second, all TB patients with DST results were a minority of the total TB patients; therefore, the observations reported in our study may not be applicable to the general TB population in China. Third, DST for second-line anti-TB drugs was not routinely carried out in Shandong, China; thus, whether diabetes had an influence on primary resistance of second-line anti-TB drugs remained a question.

\section{Conclusion}

Few previous published studies have examined the risk factors and primary drug-resistant profile of TB-DM cases. Our study indicated that TB-DM groups $(21.83 \%)$ had a higher proportion of drug resistance than TB groups $(16.96 \%)$, and diabetes was identified as a risk factor of total DR, PDR, SM resistance, and $\mathrm{INH}+\mathrm{SM}$ resistance among newly diagnosed TB cases. Further longitudinal studies exploring the short-term and long-term effects of diabetes on the transmission of resistant TB strains may be urgently needed. Our findings highlight that good management of diabetes and TB infection screening program among DM patients might be necessary for improving DR-TB control in China.

\section{Acknowledgments}

We thank Shandong Provincial Hospital, Shandong Provincial Chest Hospital, 13 Municipal-Level and 21 County-Level Local Health Departments for drug susceptibility data, sociodemographic data, and clinical data. This work was supported by the Department of Science \& Technology of Shandong Province (CN) (No. 2007GG30002033; No. 2017GSF218052) and Jinan Science and Technology Bureau (CN) (No. 201704100)

\section{Author contributions}

All authors contributed to data analysis, drafting or revising the article, gave final approval of the version to be published, and agree to be accountable for all aspects of the work.

\section{Disclosure}

The authors report no conflicts of interest in this work.

\section{References}

1. World Health Organization. Global tuberculosis report 2018; 2018 [cited December 11, 2018]. Available from: https://www.who.int/tb/ publications/global_report. Accessed July 19, 2017

2. World health statistics 2018. Available from: http://apps.who.int/iris/ bitstream/handle/10665/272596/9789241565585-eng.pdf?ua=1. Accessed July 19, 2017

3. Restrepo BI. Diabetes and Tuberculosis[M]//Understanding the Host Immune Response against Mycobacterium Tuberculosis Infection. Cham: Springer; 2018:1-21.

4. Ugarte-Gil C, Alisjahbana B, Ronacher K, et al. Diabetes mellitus among pulmonary tuberculosis patients from four TB-endemic countries: the TANDEM study. Clin Infect Dis. 2019. doi:10.1093/cid/ciz284

5. Golub JE, Mok Y, Hong S, et al. Diabetes mellitus and tuberculosis in Korean adults: impact on tuberculosis incidence, recurrence and mortality. Int $J$ Tuberc Lung Dis. 2019;23(4):507-513. doi:10.5588/ ijtld. 18.0103

6. Critchley JA, Restrepo BI, Ronacher K, et al. Defining a research agenda to address the converging epidemics of tuberculosis and diabetes: part 1: epidemiology and clinical management. Chest. 2017;152(1):165-173. doi:10.1016/j.chest.2017.04.155

7. Gandhi NR, Nunn P, Dheda K, et al. Multidrug-resistant and extensively drug-resistant tuberculosis: a threat to global control of tuberculosis. Lancet. 2010;375(9728):1830-1843. doi:10.1016/ S0140-6736(10)60410-2

8. Yang C, Luo T, Shen X, et al. Transmission of multidrug-resistant Mycobacterium tuberculosis in Shanghai, China: a retrospective observational study using whole-genome sequencing and epidemiological investigation. Lancet Infect Dis. 2017;17(3):275-284. doi:10.1016/S1473-3099(16)30418-2

9. Magee MJ, Bloss E, Shin SS, et al. Clinical characteristics, drug resistance, and treatment outcomes among tuberculosis patients with diabetes in Peru. Int $J$ Infect Dis. 2013;17(6):e404-e412. doi:10.1016/j.ijid.2012.12.029

10. Perez-Navarro LM, Restrepo BI, Fuentes-Dominguez FJ, et al. The effect size of type 2 diabetes mellitus on tuberculosis drug resistance and adverse treatment outcomes. Tuberculosis. 2017;103:83-91. doi:10.1016/j.tube.2017.01.006

11. Liu Q, Li W, Xue M, et al. Diabetes mellitus and the risk of multidrug resistant tuberculosis: a meta-analysis. Sci Rep. 2017;7(1):1090. doi:10.1038/s41598-017-01213-5

12. Wang L, Zhang H, Ruan Y, et al. Tuberculosis prevalence in China, 1990-2010; a longitudinal analysis of national survey data. Lancet. 2014;383(9934):2057-2064. doi:10.1016/S0140-6736(13)62639-2

13. Yang F, Qian D, Chen J, et al. Prevalence, awareness, treatment and control of diabetes mellitus in rural China: results from Shandong Province. Diabetic Med. 2016;33(4):454-458. doi:10.1111/dme.12842

14. Zhao Y, Zhao B, Xu S, et al. National survey of drug-resistant tuberculosis in China. $N$ Engl $J$ Med. 2012;366(23):2161-2170. doi:10.1056/NEJMoa1108789

15. El Amin NM, Hanson HS, Pettersson B, Petrini B, Von Stedingk LV. Identification of non-tuberculous mycobacteria: 16S rRNA gene sequenceanalysis vs. conventional methods. Scand J Infect Dis. 2000;32:47-50.

16. Jing $\mathrm{H}$, Wang $\mathrm{H}$, Wang $\mathrm{Y}$, et al. Prevalence of nontuberculous mycobacteria infection, China, 2004-2009. Emerg Infect Dis. 2012;18(3):527-528. doi:10.3201/eid1803.110175

17. American Diabetes Association. Diagnosis and classification of diabetes mellitus. Diabetes Care. 2010;33(Supplement 1):S62-S69. doi:10.2337/dc10-S062

18. Type 2 diabetes ADA diagnosis criteria-medscape (eMedicine), Available from: https://emedicine.medscape.com/article/2172154overview. Accessed July 19, 2017 
19. Van Rie A, Warren R, Richardson M, et al. Classification of drugresistant tuberculosis in an epidemic area. Lancet. 2000;356 (9223):22-25. doi:10.1016/S0140-6736(00)02429-6

20. Falzon D, Schünemann HJ, Harausz E, et al. World Health Organization treatment guidelines for drug-resistant tuberculosis, 2016 update. Eur Respir J. 2017;49(3):1602308. doi:10.1183/ 13993003.02308-2016

21. McMurry HS, Mendenhall E, Rajendrakumar A, et al. Coprevalence of type 2 diabetes mellitus and tuberculosis in low-income and middle-income countries: a systematic review. Diabetes Metab Res Rev. 2019;35(1):e3066. doi:10.1002/dmrr.3066

22. Kumar Nathella P, Babu S. Influence of diabetes mellitus on immunity to human tuberculosis. Immunology. 2017;152(1):13-24. doi:10.1111/imm.12762

23. Lachmandas E, Van Den Heuvel CN, Damen AM, et al. Diabetes mellitus and increased tuberculosis susceptibility: the role of shortchain fatty acids. $J$ Diabetes Res. 2016;2016:6014631-15. doi: $10.1155 / 2016 / 6014631$

24. Dobler CC, Flack JR, Marks GB. Risk of tuberculosis among people with diabetes mellitus: an Australian nationwide cohort study. BMJ Open. 2012;2(1):e000666. doi:10.1136/bmjopen-2011-000666

25. S C P, C C K, Kao D, et al. Effect of diabetes on tuberculosis control in 13 countries with high tuberculosis: a modelling study. Lancet Diabetes Endocrinol. 2015;3(5):323-330. doi:10.1016/S2213-8587(15)00042-X

26. Wu Z, Guo J, Huang Y, et al. Diabetes mellitus in patients with pulmonary tuberculosis in an aging population in Shanghai, China: prevalence, clinical characteristics and outcomes. $J$ Diabetes Complications. 2016;30(2):237-241. doi:10.1016/j.jdiacomp.2015.11.014

27. Abdullah A, Peeters A, de Courten M, Stoelwinder J. The magnitude of association between overweight and obesity and the risk of diabetes: a meta-analysis of prospective cohort studies. Diabetes Res Clin Pract. 2010;89(3):309-319. doi:10.1016/j.diabres.2010.04.012

28. Faurholt-Jepsen D, Range N, PrayGod G, et al. The role of anthropometric and other predictors for diabetes among urban Tanzanians with tuberculosis. Int J Tuberc Lung Dis. 2012;16(12):1680-1685. doi:10.5588/ijtld. 12.0360
29. Magee MJ, Kempker RR, Kipiani M, et al. Diabetes mellitus is associated with cavities, smear grade, and multidrug-resistant tuberculosis in Georgia. Int J Tuberc Lung Dis. 2015;19(6):685-692. doi: $10.5588 /$ ijtld.14.0811

30. W B L, Zhang YQ, Xing J, et al. Factors associated with primary transmission of multidrug-resistant tuberculosis compared with healthy controls in Henan Province, China. Infect Dis Poverty. 2015;4(1):14. doi:10.1186/s40249-015-0045-1

31. Gagneux S. Fitness cost of drug resistance in Mycobacterium tuberculosis. Clin Microbiol Infect. 2009;15:66-68. doi:10.1111/j.14690691.2008.02685.x

32. Grandjean L, Gilman RH, Martin L, et al. Transmission of multidrugresistant and drug-susceptible tuberculosis within households: a prospective cohort study. PLoS Med. 2015;12(6):e1001843. doi:10.1371/ journal.pmed.1001809

33. Fisher-Hoch SP, Whitney E, McCormick JB, et al. Type 2 diabetes and multidrug-resistant tuberculosis. Scand J Infect Dis. 2008;40(1112):888-893. doi:10.1080/00365540802342372

34. Gagneux S, Burgos MV, DeRiemer K, et al. Impact of bacterial genetics on the transmission of isoniazid-resistant Mycobacterium tuberculosis. PLoS Pathog. 2006;2(6):e61. doi:10.1371/journal. ppat.0020061

35. Mitchison DA. Tubercle bacilli resistant to isoniazid; virulence and response to treatment with isoniazid in guinea-pigs. $B M J$. 1954;1:128-130. doi:10.1136/bmj.1.4854.128

36. Middlebrook G, Cohn ML. Some observations on the pathogenicity of isoniazid-resistant variants of tubercle bacilli. Science. 1953;118:297-299. doi:10.1126/science.118.3063.297

37. Van Soolingen D, Borgdorff MW, de Haas PE, et al. Molecular epidemiology of tuberculosis in the Netherlands: a nationwide study from 1993 through 1997. J Infect Dis. 1999;180:726-736. doi: $10.1086 / 314930$

38. Carey IM, Critchley JA, DeWilde S, et al. Risk of infection in type 1 and type 2 diabetes compared with the general population: a matched cohort study. Diabetes Care. 2018;41(3):513-521. doi:10.2337/dc172131 


\section{Supplementary material}

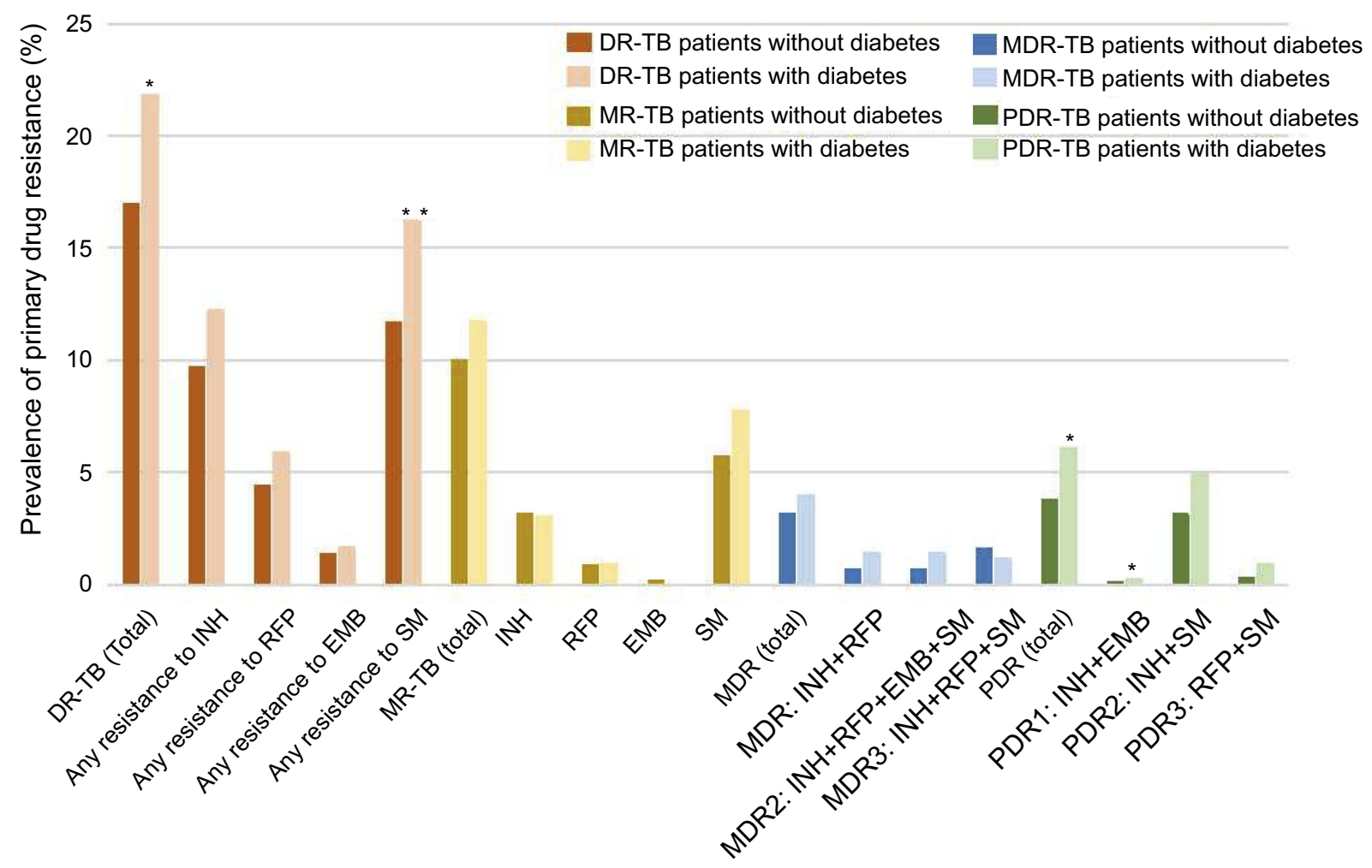

Figure SI Overall drug resistance (\%) in TB patients with and without diabetes. $* P<0.05 ; * * P<0.0$ I.

Notes: Multidrug resistance (MDR) refers to resistance to at least both isoniazide and rifampicin. MDRI refers to resistance to both isoniazide and rifampicin, but sensitive to other anti-TB drugs. MDR2 refers to resistance to isoniazide, rifampicin, ethambutol, and streptomycin, but sensitive to other anti-TB drugs. MDR3 refers to resistance to isoniazide, rifampicin, and streptomycin, but sensitive to other anti-TB drugs. Polydrug resistance (PDR) refers to resistance to more than one first-line anti-TB drug, other than both isoniazid and rifampicin. PDRI refers to resistance to isoniazide and ethambutol, but sensitive to other anti-TB drugs. PDR2 refers to resistance to both isoniazide and streptomycin, but sensitive to other anti-TB drugs. PDR3 refers to resistance to both rifampicin and streptomycin, but sensitive to other anti-TB drugs.

Abbreviations: TB, tuberculosis; DR-TB, drug-resistant tuberculosis; MR-TB, monoresistant tuberculosis; MDR-TB, multiresistant tuberculosis; PDR-TB, polydrug resistant tuberculosis; EMB, ethambutol; INH, isoniazid; RFP, rifampin; SM, streptomycin.

\section{Publish your work in this journal}

Infection and Drug Resistance is an international, peer-reviewed openaccess journal that focuses on the optimal treatment of infection (bacterial, fungal and viral) and the development and institution of preventive strategies to minimize the development and spread of resistance. The journal is specifically concerned with the epidemiology of antibiotic resistance and the mechanisms of resistance development and diffusion in both hospitals and the community. The manuscript management system is completely online and includes a very quick and fair peerreview system, which is all easy to use. Visit http://www.dovepress.com/ testimonials.php to read real quotes from published authors. 